\title{
ORIGINAL
}

\section{PREVALENCIA DE INFECCIÓN TUBERCULOSA EN LAS PERSONAS INMIGRANTES DEL ÁREA DE SALUD DE TOLEDO}

Francisco Javier Alonso Moreno (1), M. Carmen García Bajo (2), M. José Lougedo Calderón (1), José Manuel Comas Samper (3), María García Palencia (1), Francisco López de Castro(4), Noemí Pérez Villaverde (5), Reyes Sánchez García (6) y Manuel Soto García (7)

(1) Centro de Salud de Ocaña. Toledo.

(2) Centro de Salud de Sonseca. (Toledo.

(3) Centro de Salud de La Puebla de Montalbán. Toledo.

(4) Unidad Docente de Medicina Familiar y Comunitaria. Toledo.

(5) Centro de Salud de Palomarejos. Toledo.

(6) Centro de Salud de Los Yébenes. Toledo.

(7) Centro de Salud de Mora. Toledo.

\section{RESUMEN}

Fundamento: El aumento en la frecuencia de tuberculosis (TB) se produce por varios factores: resistencias al tratamiento, la infección por el virus de la inmunodeficiencia humana, el paro, la pobreza y los movimientos migratorios. El objetivo del estudio fue conocer la prevalencia de infección tuberculosa en población inmigrante del Área de Salud de Toledo.

Métodos: Estudio descriptivo, transversal y multicéntrico con emplazamiento en Atención Primaria. La captación y reclutamiento de las personas inmigrantes se realizó sobre base de tarjeta sanitaria, entre diciembre de 2002 y septiembre de 2003. Se realizó intradermorreacción de Mantoux (IDRM), con 0,1 ml (2UT) de PPD RT-23, previa firma del consentimiento informado. Otras variables recogidas fueron: edad, sexo, peso, país de origen, situación laboral, vacunación BCG, número de convivientes, tiempo de residencia en Europa.

Resultados: Fueron incluidos 344 inmigrantes, con una edad media de $28,8 \pm 12,7$ años. El 50\% (172) eran mujeres. Llevaban un promedio de 3,4 3,7 años de residencia en Europa. El 78,8\% (271) de promedio de $3,4 \pm 3,7$ años de residencia en Europa. El $78,8 \%$ (271) de tiva en 75 de ellos $[27,7 \%$ (IC 95\%: 23,1-32,5)]. Los inmigrantes con Mantoux positivo tenían más edad $(32,5 \pm 8,5$ años) frente a $(27,7 \pm$ 13,8 años) en los que el resultado fue negativo, $p=0,006$. El origen subsahariano aumentaba la probabilidad de presentar IDRM positiva.

Conclusiones: La población inmigrante del Área de Salud de Toledo presenta una prevalencia alta de infección tuberculosa, superior a la población general española. Existen diferencias según el rior a la población general española. Existen diferencias según el país de origen. Estos resultados indican la importancia de considerar
la prueba de Mantoux en las actividades preventivas dirigidas a la población inmigrante.

Palabras clave: Tuberculosis. Inmigración. Atención primaria de salud.

Correspondencia:

Francisco Javier Alonso Moreno

Centro de Salud

C/ Villasante, $\mathrm{S} / \mathrm{N}$

Ocaña

45300 Toledo

Correo electrónico: falonsom@telefonica.net
ABSTRACT

\section{Prevalence of Tuberculosis Infection among Immigrants in the Toledo Health District, Spain}

Background: The rise in the frequency of tuberculosis is due to several factors: resistance to treatment, human immunodeficiency virus infection, unemployment, poverty and migratory movements. This study is aimed at ascertaining the prevalence of tuberculosi infection among the immigrant population in the Toledo Health District.

Methods: Descriptive, cross-sectional, multi-center study conducted at the Primary Care level. The immigrants were attracted and recruited on the based of their health cards within the December 2002 -September 2003 period. Mantoux intradermal reaction test wa performed with $0.1 \mathrm{ml}$ (2UT) of PPD RT-23, following informed
consent. Other variables were collected: age, sex, weight, country of consent. Other variables were collected: age, sex, weight, country of
origin, work situation, BCG vaccination, number of individuals origin, work situation, BCG vaccination, number
living with them, length of time residing in Europe.

Results: A total of 344 immigrants were included in the study, averaging $28.8 \pm 12.7$ years of age. Fifty percent were females (172) They had been living in Europe for an average of $3.4 \pm 3.7$ years. A total $78.8 \%$ (271) of the immigrants completed the study. The intradermal reaction was positive in 75 of them $[27.7 \%$ (CI 95\%: 23.dermal reaction was positive in 75 of them $[27.7 \%$ (CI 95\%: 23 .-
$32.5)]$. Those immigrants showing a positive Mantoux were older $32.5)$ ]. Those immigrants showing a positive Mantoux were older
(age $32.5 \pm 8.5$ years) than those showing a negative result (age 27.7 (age $32.5 \pm 8.5$ years) than those showing a negative result (age 27.7
\pm 13.8 years), $p=0.006$. Originally being from the sub-Saharan area \pm 13.8 years), $\mathrm{p}=0.006$. Originally being from the sub-Saharan area
increased the probability of showing a positive intradermal reaction.

Conclusions: The immigrant population of the Toledo Health District shows a high prevalence of tuberculosis infection, which is higher than the general Spanish population. Differences exist depending upon the country of origin. These results indicate the importance of considering the Mantoux test among the preventive importance of considering the Mantoux test among the p
activities addressed toward this immigrant population.

Key words: Tuberculosis. Immigration. Primary health care. 


\section{INTRODUCCIÓN}

La tuberculosis (TB) es una enfermedad infecciosa que constituye un importante problema de salud pública por la gran morbimortalidad que produce. El reservorio principal de la TB es el individuo infectado que puede transformarse en fuente de infección si desarrolla la enfermedad y se convierte en bacilífero. La forma más común de transmisión de la TB es la aérea, por exposición al bacilo a través de aerosoles con origen en enfermos tuberculosos (tos, estornudo, hablar, etc.). La probabilidad de la infección está relacionada con la intensidad de la exposición y la efectividad de las defensas del huésped ${ }^{1}$.

En las últimas décadas se viene observando en los países desarrollados un incremento de la incidencia de casos de tuberculosis. En España se estima que cada año se producen entre 15.600 y 17.500 nuevos casos de TB (40-45/100.000 habitantes), aunque sólo se contabilizan la mitad al existir una importante infradeclaración. Nuestras tasas son muy superiores a las del resto de países desarrollados y esto se debe a que el control de la TB ha sido inadecuado ${ }^{2}$.

Este aumento en la frecuencia de TB se produce por varios factores: la aparición de resistencias al tratamiento, la relación de la TB con el virus de la inmunodeficiencia humana (VIH) y circunstancias sociodemográficas como el paro, la pobreza o los movimientos migratorios (sobre todo los procedentes de países con elevadas tasas de incidencia) $)^{3,4}$. Según datos aportados en el XXXVII Congreso de la Sociedad Española de Aparato Respiratorio (SEPAR), celebrado en Madrid en junio de 2004, entre el 25\% y el 34\% de los casos de TB en nuestro país son personas inmigrantes. En el Hospital Ramón y Cajal de Madrid el 3,9\% de las personas adultas inmigrantes atendidas por cualquier patología, padecía una TB activa ${ }^{5}$.

En efecto, algunas comunidades de inmigrantes presentan tasas elevadas de infec- ción tuberculosa, sobretodo los originarios del África Subsahariana, Europa del Este y Asia ${ }^{6,7}$. Todo ello se debe, entre otras causas, a las deficientes condiciones de vivienda y a las características de los agrupamientos (hacinamientos, agrupaciones no familiares con frecuentes cambios de domicilio y de compañeros de vivienda) que provocan mayor prevalencia de enfermedades infecciosas $^{8}$.

Esto hace que las personas inmigrantes sean un grupo prioritario para una vigilancia y control de la infección tuberculosa, ya que representan un colectivo de población joven y con una alta prevalencia de esta infección. En consecuencia, se recomienda realizar un cribado de la enfermedad tuberculosa a cualquier persona inmigrante ${ }^{9}$. Es aconsejable, al menos, la realización de la prueba de la tuberculina a los menores de 35 años, en los que la profilaxis con isoniacida puede implantarse con bajo riesgo de toxicidad (durante 6 meses) ${ }^{10}$.

Por todo ello, consideramos importante realizar el presente estudio, cuyo objetivo fue conocer la prevalencia de infección TB en población inmigrante del Área de Salud de Toledo.

\section{SUJETOS Y MÉTODOS}

Se realizó un estudio descriptivo, transversal y multicéntrico en población inmigrante seguida en el ámbito de la Atención Primaria. El trabajo de campo se realizó entre diciembre de 2002 y septiembre de 2003, como un proyecto de investigación de un equipo multidisciplinario integrado por médicos, enfermeros y trabajadores sociales del Área de Salud de Toledo.

La captación y reclutamiento se hizo en base al registro de tarjeta sanitaria, entre diciembre de 2002 y septiembre de 2003. Los trabajadores sociales informaron del estudio y pidieron su libre participación a las 
personas inmigrantes que habían solicitado su tarjeta y a los mediadores comunitarios, inmigrantes que conocen nuestro idioma y actuaban facilitando la comunicación con estas personas.

Los criterios de inclusión del estudio fueron: inmigrantes que aceptaron participar en el mismo con domicilio en las zonas de salud de Añover de Tajo, Fuensalida, La Puebla de Montalbán, Ocaña, Sonseca y Villaluenga. Fueron excluidos aquéllos que presentaron enfermedad aguda que impidió la asistencia al Centro de Salud y por tanto la participación en el estudio.

\section{Datos de las personas inmigrantes}

Fueron registradas la edad, sexo, peso, procedencia (país de origen), zona de salud, situación laboral, vacunación BCG, número de convivientes, tiempo de residencia en Europa.

\section{Prueba de Mantoux}

La prueba normalizada por la Organización Mundial de la Salud (OMS) y admitida para uso general es la intradermorreacción de Mantoux (IDRM). La dosis administrada fue de 0,1 ml (2UT) de PPD RT-23, que se inyectó intradérmicamente en la cara ante- rior del antebrazo, traccionando la piel con la mano contraria a la que sujeta la jeringa. La aguja se debe colocar tangencial a la dermis y con el bisel hacia arriba (aguja de acero corta y biselada del calibre 27). Se debía formar una pápula de 6 a $10 \mathrm{~mm}$ de diámetro. Se marcó con un círculo la zona de la pápula y se recomendó no friccionarla. $\mathrm{La}$ lectura de la IDRM se realizó entre las 48 y 72 horas. Sólo se midió la induración y no el eritema producido, considerando el diámetro de la induración transversal al eje mayor del antebrazo. Los criterios de positividad de la IDRM se especifican en la tabla 1.

Se solicitó firma del consentimiento informado.

Los profesionales de los centros de salud que participaron en el estudio recibieron información para descartar la presencia casos de TB activa y administración de quimioprofilaxis.

\section{Análisis estadístico}

La descripción de las variables se hizo mediante los parámetros habituales (porcentaje, media, desviación típica, etc.), con los correspondientes intervalos de confianza (IC) del 95\%. En el análisis se emplearon los tests de la $\chi^{2}$ de Pearson, para comparación de porcentajes, y la t de Student y análisis de

Tabla 1

Interpretación de la intradermorreacción de Mantoux. (Criterios basados en: Targeted tuberculin testing and treatment of latent tuberculosis infection ${ }^{1}$ )

\begin{tabular}{|c|c|}
\hline Positividad del test de Mantoux & Condiciones \\
\hline$\geq 10 \mathrm{~mm}$ & $\begin{array}{l}\text { - Inmigrantes que residan en el país por un periodo } \leq 5 \text { años } \\
\text {-Niños }<4 \text { años } \\
\text { - Niños y adolescentes convivientes con adultos de riesgo } \\
\text { - Adictos a drogas por vía intravenosa } \\
\text { - Trabajadores sanitarios, personal de laboratorio } \\
\text { - Otras enfermedades o condiciones de riesgo }\end{array}$ \\
\hline$\geq 15 \mathrm{~mm}$ & $\begin{array}{l}\text {-Inmigrantes residentes en el país por un periodo }>\text { 5años } \\
\text {-El resto }\end{array}$ \\
\hline
\end{tabular}

HIV: virus de la inmunodeficiencia humana, SIDA: síndrome de la inmunodeficiencia adquirida 
varianza (ANOVA), para comparación de medias y varianzas. Se realizó también una regresión logística no condicional por el método stepwise backward (LR). Las variables independientes incluidas en el modelo fueron la edad, sexo, peso y origen subsahariano, mientras que la variable dependiente fue la IDRM positiva.

\section{RESULTADOS}

De las 458 personas inmigrantes contactadas, 114 no acudieron a la cita. Fueron incluidos en el estudio las 344 restantes. Su edad media era de 28,8 años ( $\mathrm{DE}=12,7$; rango de 0 a 81 años) y el 50,0 \% (172) eran mujeres. La edad de los hombres $(29,7 \pm 12,1$ años) fue ligeramente mayor que la de las mujeres $(27,9 \pm 13,4$ años). Llevaban un promedio de 3,4 años $(\mathrm{DE}=3,7)$ de residencia en Europa.

De los 344 sujetos completaron el estudio $271(78,8 \%) ; 44(12,8 \%)$ no acudieron a la lectura y en 29 mujeres $(8,4 \%)$ no se realizó la prueba por embarazo o lactancia. La IDRM fue positiva en 75 de ellos $[27,7 \%$ (IC $95 \% ; 23,1-32,5)]$.
La prevalencia de infección tuberculosa por grupos de edad se muestra en la tabla 2, en la que puede apreciarse cómo la mayor frecuencia $(38,7 \%)$ apareció en el grupo de edad 30-39 años. Los sujetos con Mantoux positivo tenían más edad (32,5 $\pm 8,5$ años) frente a aquéllos los que el resultado fue negativo, $(27,7 \pm 13,8$ años $)(p=0,006)$.

En la misma tabla puede observarse también una mayor frecuencia de IDRM positiva en hombres $(30,7 \%)$ y en las personas inmigrantes con tres o más años residencia en nuestro país $(33,3 \%)$.

La prevalencia de infección tuberculosa fue diferente en las distintas zonas de salud del área $\left(\chi^{2}=26,77 ; p=0,001\right)$ (figura 1). Según el país de origen, la mayor prevalencia correspondió a los naturales de Malí $(45,1 \%)$ y Pakistán $(38,4 \%)$ (figura 2). Después de realizar un ajuste simultáneo de las distintas variables incluidas en el estudio, se observó que el origen subsahariano aumentaba la probabilidad de presentar IDRM positiva; odds ratio [2,05 (IC 95\%: 1,13,83)] (tabla 3).

Tabla 2

Prevalencia de infección tuberculosa por grupos de edad en población inmigrante (Toledo, 2002-2003)

\begin{tabular}{|c|c|c|c|}
\hline Grupo de edad & Positivos / Inmigrantes & Porcentaje & IC 95\% \\
\hline $0-19$ & $5 / 64$ & 7,8 & $1,3-14,3$ \\
\hline $20-29$ & $21 / 62$ & 33,9 & $22,2-45,6$ \\
\hline $30-39$ & $36 / 93$ & 38,7 & $28,9-48,5$ \\
\hline Desconocida & $13 / 58$ & & \\
\hline Sexo & & & \\
\hline Hombres & $43 / 140$ & 30,7 & $23,1-38,3$ \\
\hline Mujeres & $32 / 129$ & 24,8 & $17,4-32,2$ \\
\hline Desconocido & $0 / 8$ & & \\
\hline Residencia Europa & & & \\
\hline$\geq 3$ años & $38 / 114$ & 33,3 & $24,7-41,9$ \\
\hline$<3$ años & $37 / 154$ & 24 & $17,3-30,7$ \\
\hline Desconocida & $0 / 9$ & & \\
\hline
\end{tabular}

IC: intervalo de confianza 
Figura 1

Prevalencia de infección tuberculosa según Zona de Salud (Toledo, 2002-2003)

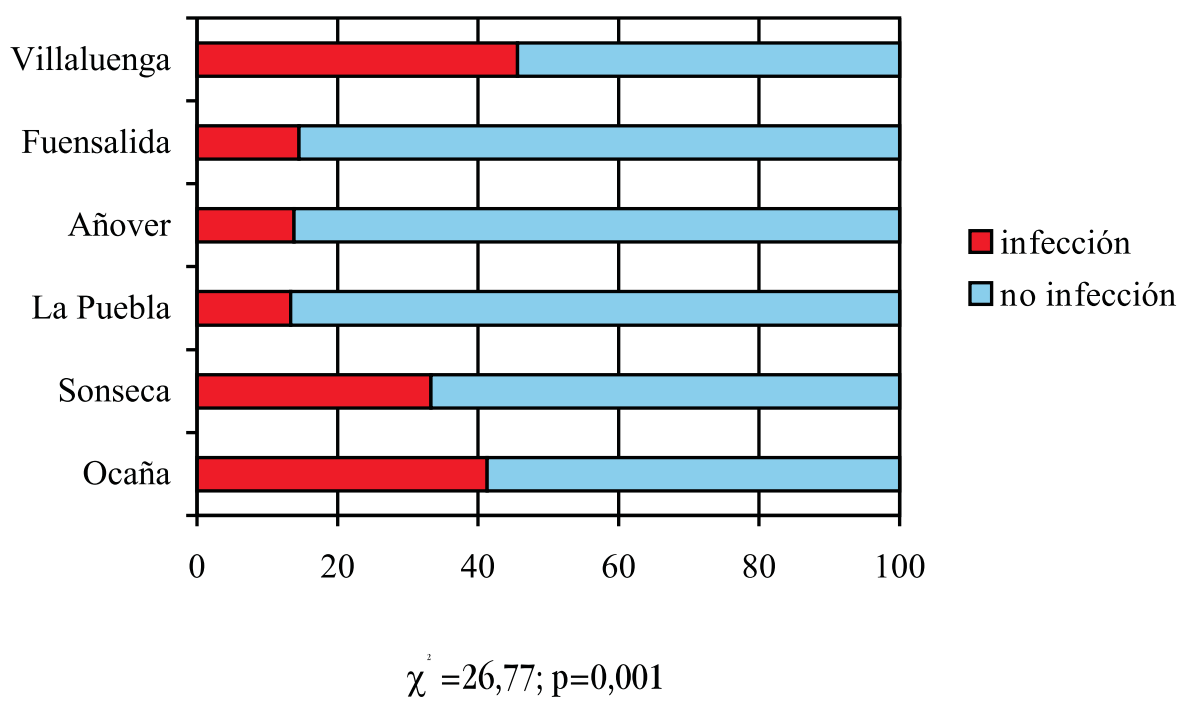

Figura 2

Prevalencia de infección tuberculosa, según país de origen (Toledo, 2002-2003)

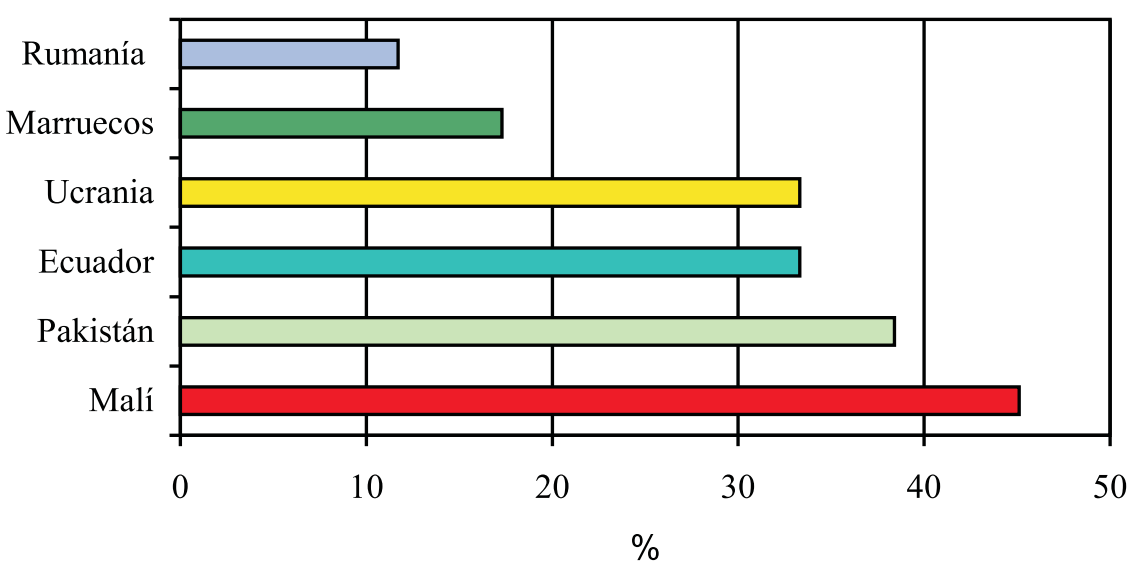

Respecto al diámetro de la IDRM, resultó significativamente mayor $(\mathrm{T}=2,974$; $\mathrm{p}=0,003$ ) en las personas inmigrantes que llevaban tres o más años de residencia en Europa $(7,03 \mathrm{~mm})$, que en los que llevaban menos $(4,5 \mathrm{~mm})$. También resultó mayor en los hombres $(6,4 \mathrm{~mm})$ que en las mujeres
(4,8 $\mathrm{mm})$, aunque las diferencias no fueron estadísticamente significativas $(\mathrm{T}=1,896$; $\mathrm{p}=0,059)$. El diámetro fue significativamente mayor $(T=5,446 ; p=0,001)$ en las personas inmigrantes de 20 ó más años $(6,87 \mathrm{~mm})$, que en las personas inmigrantes menores de 20 años (1,76 mm) (figura 3). 
Tabla 3

Resultados de la regresión logística

\begin{tabular}{|l|c|c|c|}
\hline Variables independientes & OR & IC95\% & Significación Estadística \\
\hline Origen subsahariano & 2,05 & $1,1-3,83$ & $\mathrm{p}=0,024$ \\
\hline Peso & 1,02 & $0,99-1,04$ & NS \\
\hline Edad & 1,0 & $0,96-1,03$ & NS \\
\hline Sexo & 0,8 & $0,5-1,37$ & NS \\
\hline
\end{tabular}

OR= ODSS RATIO; IC: INTERVALO DE CONFIANZA

Figura 3

Tamaño de la intradermorreacción de Mantoux según edad, años de residencia y sexo de las personas inmigrantes (Toledo, 2002-2003)

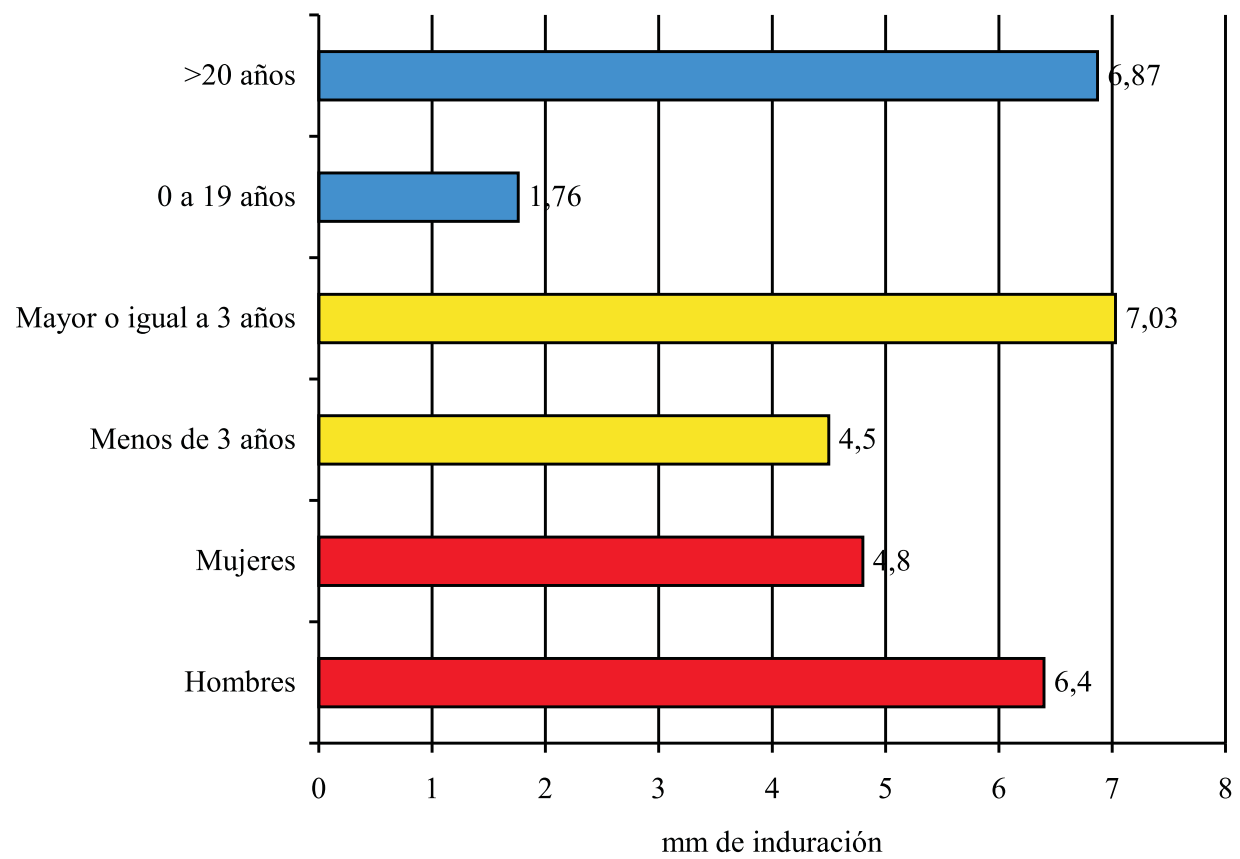

\section{DISCUSIÓN}

Una de las posibles limitaciones de este estudio fue no contar con una muestra de base poblacional. No obstante debido a la dificultad que esto supone en este tipo de población, optamos por utilizar el registro de tarjeta sanitaria que puede ser representati- vo, ya que las personas inmigrantes están autorizadas a solicitar su tarjeta sanitaria, al estar inscritas en el padrón del municipio donde residen habitualmente ${ }^{11}$.

Cerca del $80 \%$ de las personas inmigrantes acudieron a la lectura de la IDRM, resultados que podemos considerar como satis- 
Tabla 4

Prevalencia de infección tuberculosa en distintos colectivos de inmigrantes en España y Toledo

\begin{tabular}{|l|c|c|}
\hline \multicolumn{1}{|c|}{ Países } & Estudios nacionales* & Toledo \\
\hline Guinea, África subsahariana & $46,9-51,9$ & 45,1 \\
\hline Paquistán & 37,5 & 38,4 \\
\hline América latina & 25,3 & 33,3 \\
\hline Marruecos & $14-50$ & 17,3 \\
\hline Europa oriental & 42,7 & $11,7-33,3$ \\
\hline
\end{tabular}

* Adaptado de Grupo de Trabajo de los Talleres de 2001 y 2002 de la Unidad de Investigación de Tuberculosis en Barcelona. Prevención y control de las tuberculosis importadas. Med Clin (Barc) 2003; 121: 549-62.

factorios. Aunque en caso de embarazo o lactancia no estaría contraindicada la prueba de Mantoux, preferimos no someter a la misma a estas mujeres para evitar una posible reacción de rechazo en las mismas.

La prevalencia de infección tuberculosa ha resultado mayor en las personas inmigrantes de nuestra zona que en la población española no vacunada ${ }^{12}$. En ésta última la prevalencia encontrada tanto en el grupo de edad de 20 a 29 años $(12,1 \%)$, como en el de 30 a 39 años $(22,7 \%)$, está en ambos casos por debajo de los resultados de nuestro estudio. Los resultados de nuestro trabajo aportan valores en la misma línea que los obtenidos por Durán y colaboradores ${ }^{13}$ en inmigrantes recién llegados a Barcelona. Sin embargo, la prevalencia encontrada en los procedentes del Magreb fue menor en nuestro estudio que en el realizado por García$\mathrm{Vidal}^{7}$ y colaboradores, en el que aparece un $50,7 \%$ de IDRM positiva. Los resultados obtenidos en personas inmigrantes residentes en Toledo son similares a los que se presentan en los países considerados pobres ${ }^{14}$.

La tasa de infección tuberculosa en personas inmigrantes varía mucho de unos estudios a otros, posiblemente por las diferentes características de los sujetos estudiados en cada uno de ellos. En los asentamientos de Ceuta, con inmigrantes procedentes princi- palmente de países subsaharianos, la prevalencia de infección tuberculosa fue del $32,5 \%{ }^{15}$. En colectivos de inmigrantes de procedencia norteafricana residentes en la Comunidad de Madrid ${ }^{16,17}$ fue superior al $70 \%$. En nuestro estudio las personas inmigrantes subsaharianas presentaron cifras más elevadas $(45,1 \%)$, por el contrario en los inmigrantes marroquíes encontramos una menor frecuencia de infección tuberculosa $(17,3 \%)$.

En una reciente publicación ${ }^{18}$ se presentan los resultados de la prueba de tuberculina en sujetos inmigrantes residentes en España según el país de origen. En la tabla 4 se comparan algunos de estos resultados y los obtenidos en nuestro estudio.

Esto explicaría también las diferencias observadas en la prevalencia de infección tuberculosa en las distintas zonas de salud de nuestra área. En la Zona de Villaluenga la procedencia de las personas inmigrantes es mayoritariamente subsahariana, mientras que en la Zona de Sonseca predominan los de origen pakistaní. El resto de las Zonas presentan una mayor procedencia de Marruecos y Ecuador.

Como conclusión, la alta prevalencia de esta infección en determinados colectivos de sujetos inmigrantes, consideramos que justi- 
fica la realización de un examen de salud en el primer contacto con el sistema sanitario para descartarla. En atención primaria hemos de hacer un esfuerzo para captar precozmente a estas personas. Para ello no sería disparatado acercarnos a estos colectivos a través de sus líderes comunitarios y mediadores culturales.

Pensamos que debería profundizarse más en este tema, no sólo en lo referente al estudio de su prevalencia, sino en aspectos como los conocimientos, actitudes y hábitos de las personas inmigrantes hacia la tuberculosis que nos puedan ayudar a prevenir y controlar esta enfermedad.

\section{BIBLIOGRAFÍA}

1. García C, Fernández de la Hoz K, Carpintero JL, Verde C, Puchades MJ. Manual para la Prevención y Control de la Tuberculosis. Toledo: Consejería de Sanidad. Junta de Comunidades de Castilla-La Mancha; 1995.

2. VVAA. Documento de consenso sobre la prevención y control de la tuberculosis en España. Med Clin (Barc) 1999; 113: 710-5.

3. Díaz M for The MPTR Study Group. Incidence of tuberculosis in Spain: preliminary results from the Multicenter Project for TB research (MPTR). Int J Tuberc Lung Dis 1998; 2 (supl 2): 200-1.

4. Pérez Agudo F, Alonso Moreno FJ, Urbina Torija J. Prevalencia de infección por el virus de la inmunodeficiencia humana tipo 1 y de Mycobacterium tuberculosis en una población reclusa entre los años 1.989 y 1.995. Med Clin (Barc) 1.998; 110: 167-70.

5. López-Vélez R. Enfermedades infecciosas en inmigrantes (I). FMC-Formación Médica Continuada en Atención Primaria 1996; 3: 222-8

6. Lacalle M, Gil G, Sagardui JK, González E, Martínez R y Orden B. Resultados de la aplicación de un examen de salud en población inmigrante. Aten Primaria 2000; 25: 634-8.

7. García J, Jansà JM, García P, Barnés I, Caylá JA. Enfermedades infecciosas y características sociodemográficas de los emigrantes extranjeros del
Centro Penitenciario de hombres de Barcelona. Rev Esp Salud Pública 1998; 72: 197-208.

8. Grupo de Trabajo de la Subdirección Provincial de Atención Primaria. La Atención a la Población Inmigrante en el Insalud de Madrid: propuestas de mejora. Madrid: Dirección Territorial Insalud; 2001.

9. Huerga H, López-Vélez R. Examen de salud. En: Alonso A, Huerga H, Morera J. Guía de Atención al inmigrante. Madrid: Ergon Ediciones; 2003. p. $73-$ 90.

10. CDC. Tuberculosis among foreing-born persons entering United States. MMWR 1990; 39: 1-21.

11. Real Decreto $864 / 2001$ de 20 de julio por el que se aprueba el Reglamento de ejecución de la Ley Orgánica 4/2000, de 11 de enero sobre derechos y libertades de los extranjeros en España. Y su integración social reformada por la Ley Orgánica 8/2000 de 22 de diciembre (BOE 21/7/2001).

12. Prevalencia de la infección tuberculosa en adultos. Montbrió: Actas XI Reunión del Área TIR de SEPAR, 1998.

13. Durán E, Cabezos J, Ros M, Terre M, Zarzuela F, Bada JL. Tuberculosis en inmigrantes recién llegados a Barcelona. Med Clin (Barc) 1996; 106: 525 -

14. López-Vélez R, Huerga H. Inmigración y salud. Aproximación desde Atención Primaria. Madrid: PBM Ediciones; 2002.

15. Fernández MT, Díaz J, Sánchez JM, Pérez A, Vadillo J. Prevalencia de infección tuberculosa en la población de inmigrantes en Ceuta, España. Rev Esp Salud Pública 2001; 75: 551-8.

16. Rivas Clemente FP, Nacher Conches M, Corrillero Martin J, García-Herreros Madueno MT. Prevalencia de infección tuberculosa en inmigrantes magrebíes. Med Clin (Barc) 2000;114:245-9.

17. Palomo ML, Rodríguez C, Ayerbe MT. Un estudio de contactos de tuberculosis en inmigrantes marroquíes. Rev Esp Salud Pública 1996; 70: 345-8.

18. Grupo de Trabajo de los Talleres de 2001 y 2002 de la Unidad de Investigación de Tuberculosis en Barcelona. Prevención y control de las tuberculosis importadas. Med Clin (Barc) 2003; 121: 549-62.

19. Targeted tuberculin testing and treatment of latent tuberculosis infection. Am J Respir Crit Care Med 2000; 161: S221-S247. 